\title{
Remotely-controlled orthodontics: fundamentals and description of the Dental Monitoring system
}

\author{
L.-C. Roisin'1, D. Brézulier², O. Sorel ${ }^{3}$ \\ 1 Stomatologist at DFO \\ 2 Resident at DFO, University of Rennes 1, CHU Rennes \\ 3 University Professor-Hospital Practitioner, University of Rennes 1, CHU Rennes
}

\begin{abstract}
The concept of self-measurement is already extensively used in healthcare, notably to monitor diabetes or hypertension. Drawing from this model, Dental Monitoring ${ }^{T M}$ built a new three-dimensional (3D) technology to measure tooth movement from simple photographs taken from a patient's smartphone, and an initial 3D model provided by the orthodontists. This system provides precise measurements from settings outside the clinic, and in between appointments. A team of orthodontists reviews all measurements and photographs before they are updated on a web-based platform for the practitioners. A dashboard of all monitored patients offers a comprehensive solution based on a triptych: communication supervised by orthodontists with patients and the correspondents; treatment progress evaluation through photographs taken by patients; notification with alerts send to the practitioner in any case of any abnormal clinical events, so that they can take adapted decision.
\end{abstract}

KEYWORDS

Monitoring, self-measurement, remote evaluation, 3D technology, communication, compliance

\section{INTRODUCTION}

In medicine, self-measurement and monitoring have been used for many years and have become paradigms, especially in the therapeutic follow-up of diabetes $3,22,37,40,42$ and arterial hypertension 2,11,31,32,33,41. Orthodontics requires regular monitoring of the displacements, appliances, and aids.
Remote monitoring is a new possibility that meets a real need regardless of the type of technique used. The Dental Monitoring system (DMS), the only solution to our knowledge, has, for the first time ever, provided a comprehensive and structured response to this need.

\section{Address for correspondence:}




\section{THE BASICS OF MEDICAL MONITORING}

According to the relevant literature as well as the conclusions and recommendations of scientific societies, self-measurement has definitive benefits in terms of the monitoring, the results and the quality of treatment. Public health will greatly benefit from monitoring $19,24,36$.

\section{Therapeutic benefits}

\section{Improved compliance}

Therapeutic education places patients at the heart of the treatment. They become invested in their health, and more involved in the adherence to their prescriptions. Self-measurement allows this process to progress from a passive stage of being provided with information to an active stage where they involve themselves in the treatment proces ${ }^{15,17,18,25}$.

\section{Continuous treatment adaptation and optimization}

The frequent and regular measurement of the metabolic constants (blood glucose, blood pressure, etc.) makes it possible to individualize and adapt the therapeutic protocol, to avoid under-correction or over-correction, which could imply a potentially dangerous overdose $e^{28,35}$.

\section{Early detection of incidents}

Monitoring allows a type of control with an adapted frequency, incompatible with a conventional consultation. This allows early detection of an abnormal variation of the monitored constants, thus avoiding aggravations and complications.

\section{Impact on public health}

Monitoring decreases in the number of visits to the office. If self-measurement of blood glucose ${ }^{38}$ and blood pressure ${ }^{9,14}$ are considered as examples, more efficient control can be achieved with fewer number of visits to the doctor's office. This is economical, both in terms of patient displacement and the practitioner's workflow regulation.

Without replacing the value of a complete consultation with a clinical examination, numerous consultations, dedicated to the simple systematic control of metabolic constants, can thus be saved ${ }^{6}$.

\section{Reduction in the number of hospitalizations}

The detection and early correction of any abnormalities present in the monitored constants decreases the risk of cardiovascular or cerebral complications. As a result, days spent being hospitalized in intensive care (for heart attack treatment), collateral health care and possible sequelae are falling ${ }^{5,20,26}$. The associated costs are therefore decreased.

\section{A public health objective: $70 \%$ patients monitored by self-measurement}

Improved therapeutic management, coupled with public health 
savings, means that the entire scientific community, supported by leading scientific societies, health insurance agencies and government health

\section{THE ORTHODONTIC PROBLEM}

Orthodontics has similarities in the need for follow-up of patients 4,7, 12,13,16,23,29,30,34.

\section{Therapeutic benefits in DFO}

\section{The need for compliance}

Compliance is a must in orthodontics regardless of the techniques.

- Techniques using removable devices for interception, active treatment, or contention involve strict patient cooperation.

- All the fixed techniques, vestibular or lingual, require that oral hygiene be reinforced and that patients are strongly motivated to maintain it.

- Likewise, elastic bands should be worn continuously by patients, and this must be encouraged.

\section{The need for therapeutic optimization}

Nowadays, it is impossible to precisely follow orthopedic and orthodontic developments.

- The frequency with which the splints are changed in aligner treatments could be improved by following the active phases of dental displacement, because it would allow for the monitoring of the arches in fixed treatments. organizations have set the following target: $70 \%$ patients being treated for hypertension will be monitored via self-measurement.

- Remotely managing a discrepancy via intermaxillary tractions could mean the difference between removing traction if clinical progress is observed or continuing to wear them if no progress is observed.

- Wouldn't the individualization of the time spent wearing a contention splint benefit from monitoring?

\section{Incident prevention}

Incidents between appointments are frequent. They may go unnoticed or neglected by patients. These incidents can have a negative impact on health or lead to complications that may prolong the treatment duration.

- For example, the detection of gingival embedding by a Quad Helix or a transpalatal arch before the next appointment can avoid an unpleasant session.

- During multiattachment treatment, the early detection of small complications could lead to their quick resolution, whereas they can represent a loss of control if they remain unknown until the next appointment.

- During contention, many relapses, which may not be painful or severe, may go initially unnoticed. Early detection would allow for simple repair. 


\section{Economic \& health benefits}

\section{Optimizing the number of visits}

Quick and simple controls could be monitored remotely, which would decrease the commutes for the patients (and relatives) as well as the amount of time spent in the chair. Wouldn't the use of the modern means of communication (mobile phone, Internet) make it possible to decrease the number of appointments?

These controls could be easily included in orthopedic expansion when activating the actuator, in multiattachment treatments to control

DM

DM is a French start-up company founded in 2015. After $>3$ years of research and development, it offered the first solution for the remote monitoring of orthodontic treatment. The technological challenge is twofold: to develop a technology that allows us to precisely measure the dental movements occurring between two appointments on the one hand, and to transform patients' simple mobile phone into a real self-measurement tool for orthodontists.

DM has not limited itself to a simple technical self-measurement tool and has responded to a third challenge: to develop a complete service specifically dedicated to orthodontic problems,

\section{THE DM PROCEDURE}

DM provides patients with a mobile application and a specific cheek hygiene, or when wearing elastics or contention devices.

\section{Preventing complications}

The early detection of incidents by remote monitoring allows patients to be immediately contacted. This will not increase the number of hospitalizations but will serve as a quick and effective "orthodontic emergency" system. Patients will receive optimal care in a calm and serene setting in specific time slots. The near-immediate aspect of the treatment saves treatment time, which benefits all parties-patients (and their relatives), the practitioners, and health insurance agencies-in the end.

where all the results and measurements are controlled and analyzed by a team of clinicians to validate the clinical relevance of these results and to notify the practitioners. It is a complete remotely-controlled solution structured on a triptych:

- A communication service to facilitate correspondence between orthodontists, patients, and the other treatment stakeholders.

- A treatment assessment service using photos and measurement tools to check dental movements.

- A notification service to the practitioner describing the occurrence of abnormal clinical events.

retractor, which is necessary for patients when taking intraoral photographs, and 
for the calibration of dental motion calculations (Figs. 1, 2). The practitioner defines the frequency of control to suitably monitor the treatment. The recommended frequency is every 2 weeks for active treatment and every 2 months for contention. The choice of interval is a compromise, between not requesting too much of patients and not spacing out the controls to such an extent that it jeopardizes the early detection of potential incidents.

At a predetermined interval, the application will automatically remind patients to take a series of ten intraoral photographs: three in occlusion, five with the mouth slightly open, and two occlusal views. In the case of treatment by removable devices, additional views are to be provided with the device in the mouth. These photographs are automatically sent via the mobile application to the DM computing platform.

The quality of the photos is systematically and immediately controlled not only from a technical point of view but also from a clinical point of view. If an important clinical alert is detected,



Figure 1

Mobile app.

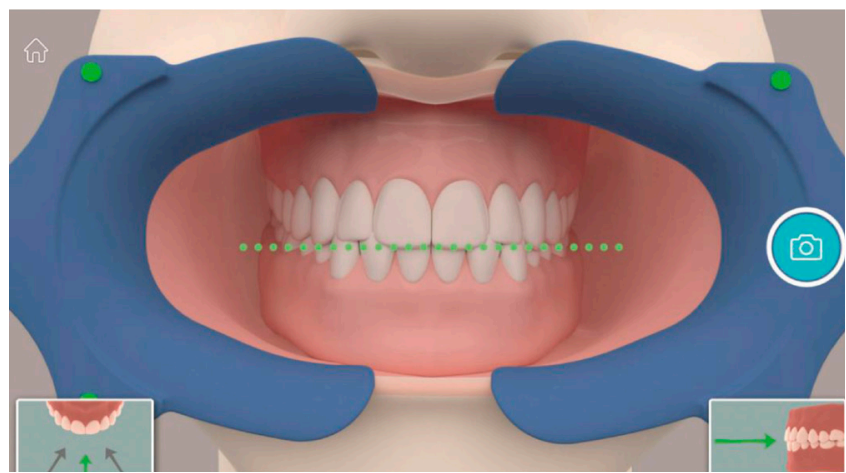

Figure 2

Specialized cheek retractor with calibration points. 
the prescribing practitioner is notified immediately, otherwise the procedure for calculating the movements is initiated and the practitioner will receive the measurement of the movements and the clinical report at the same time.

On one hand, algorithms of image analysis and optimization are used and information cards are produced from the series of photographs sent by the mobile application. On the other hand, the precise morphological measurement of the teeth resulting from the initial 3D model of patients' arches in occlusion will permit the calculation of a revised version of the 3D model of the arches $1,10,21,27,39$.

The measurement of the movements is thus established very precisely, 0.05 $\mathrm{mm}$ on the anterior teeth and $0.07 \mathrm{~mm}$

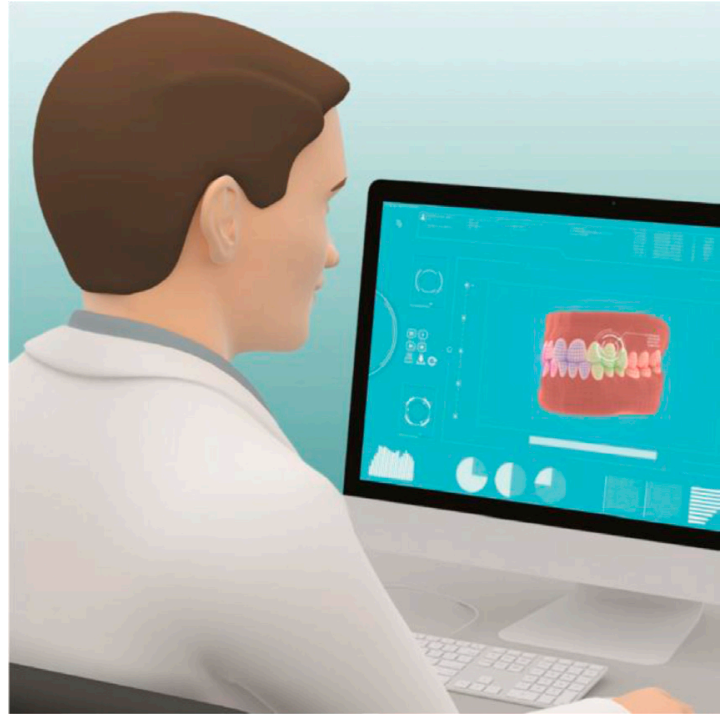

Figure 3

Results on the dashboard.

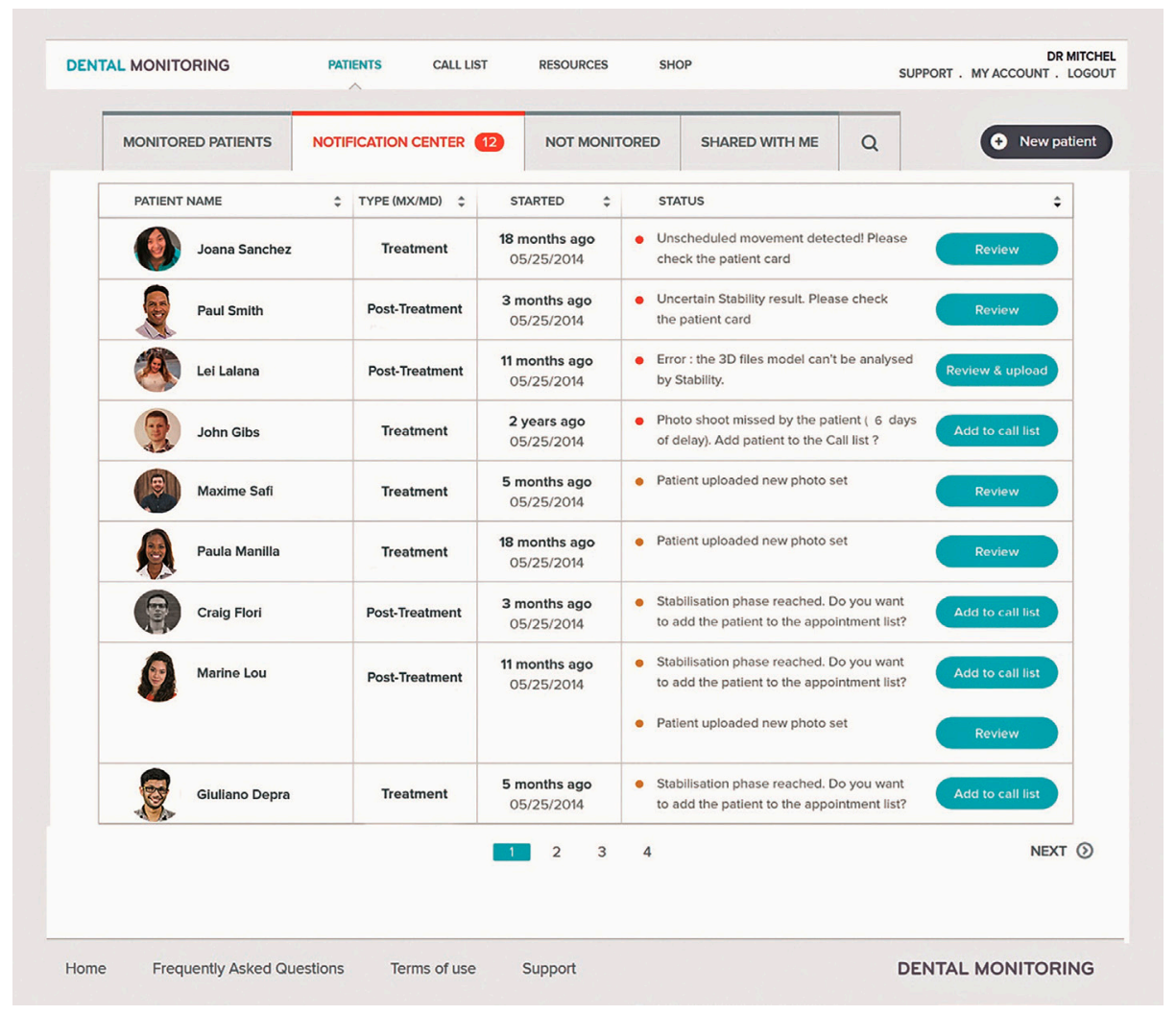

Figure 4

Web service with a list of patients being monitored. 
on the posterior teeth. When the information received does not permit measurement, the prescribing practitioner is notified on his dashboard.

All the results, photographs and measurements of the movements are then checked by a member of the DM orthodontic team who will report, if necessary, any incident or abnormal movement. The monitored patients' results are then published to the dashboard via an online platform (Figs. 3, 4).

\section{DM TOOLS}

For each patient, there are several tools at orthodontists' disposal (Fig. 5).

\section{Photo gallery}

A complete gallery of 10 photos, taken every fortnight during a treatment, allows an evaluation of the clinical evolution which is very meticulously documented.

\section{Activity curve}

Two curves, one maxillary and the other mandibular, measure the average of the sum of the movements of each tooth, from the initial model. This gives a global view of the average activity of the arch movements.

During treatment, an upward slope reflects an active device, a flat slope reflects a stabilization phase, and a downward slope is linked to a relapse in the movements.

When attaching the restraints in contention, a flat slope reflects the stability of the result while an upward slope is a sign of relapse. A downward slope marks the correction of the relapse.

\section{Detailed curves}

For each tooth, a graph plots the evolution of movements in three axes of translation and three axes of

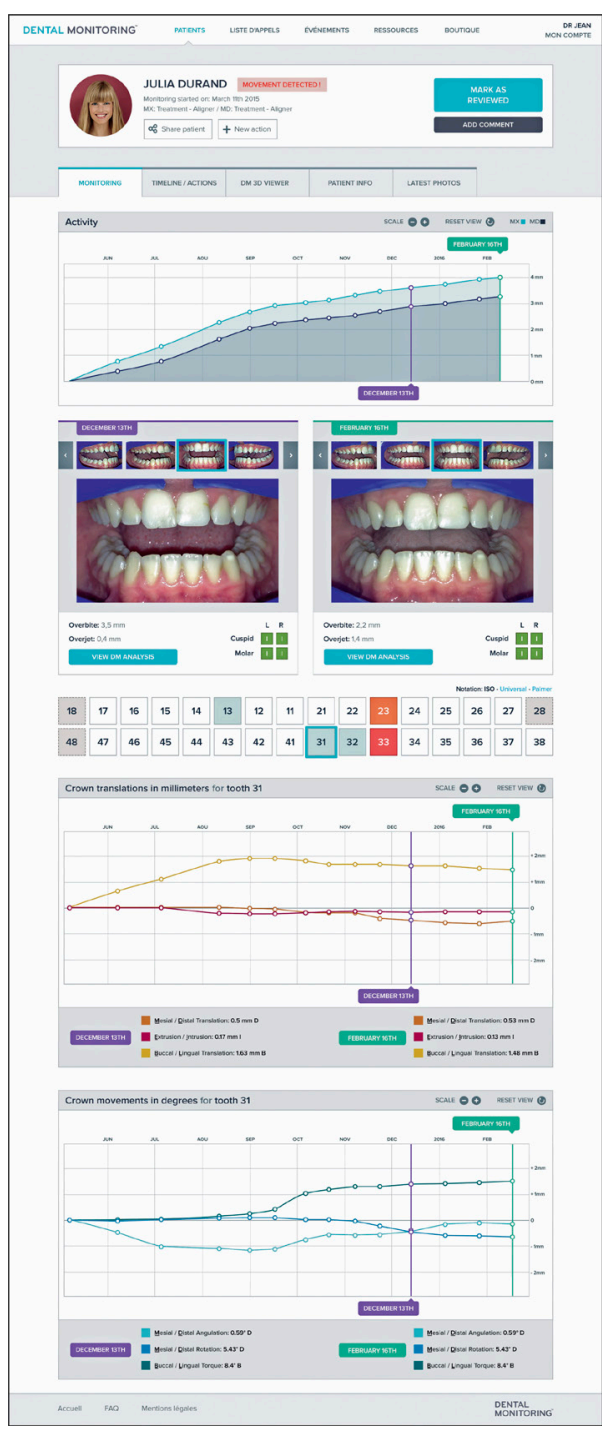

Figure 5

Patient sheet with activity curve, photo gallery, detailed curves per tooth. 
rotation-i.e., nearly 168 measurements for each photo examination of patients (mesial/distal translation, extrusion/intrusion, buccal/lingual translation, angulation, mesial/distal rotation, and vestibular/lingual torque).

\section{D matching}

3D matching allows us to superimpose the teeth of each of the 3D models calculated on previous dates. It is the visual expression of all the movements that can be found on the detailed graphs of each tooth.

In a very complementary way to the commonly used methods of treatment plan simulation, 3D matching is one of the first 3D visualization tools of real movements. 3D Matching has two main objectives:

- To facilitate, in time and space, the practitioners' analysis of all dental movements.
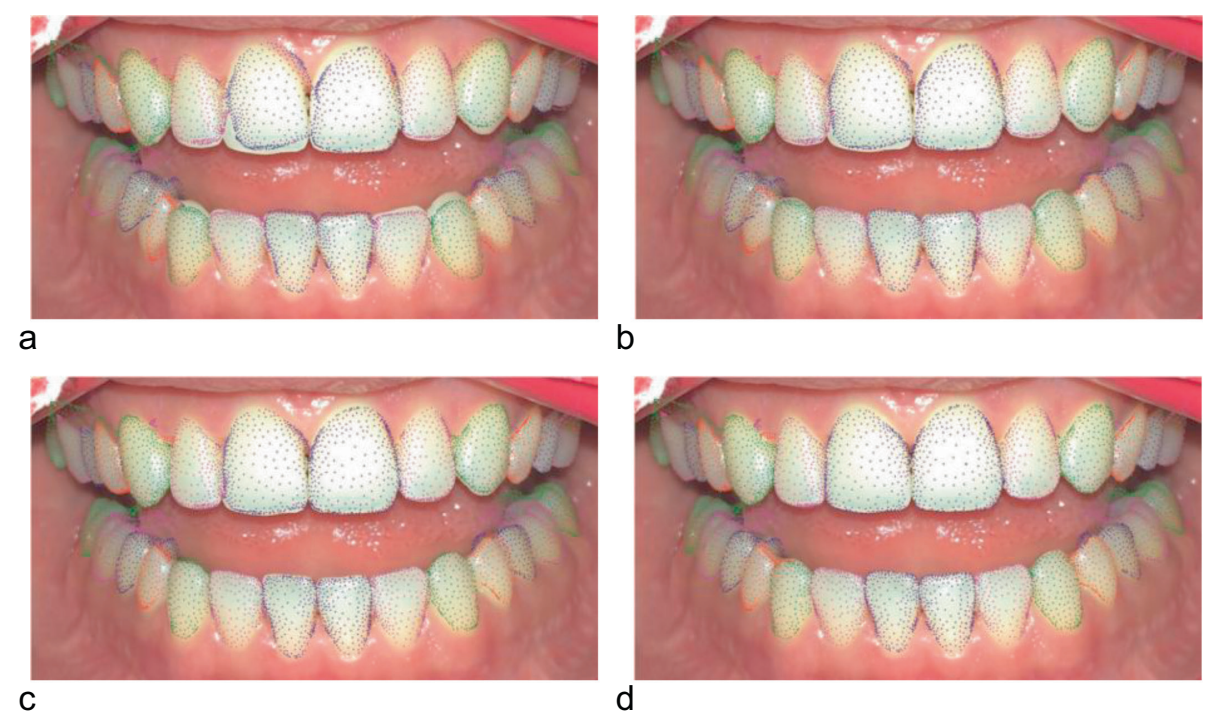

Figure $6 a, b, c, d$

$3 D$ matching makes it possible to visualize in actual photography the actual evolution of previous movements. 


\section{Patient communication tools}

Patients can access the photo history taken on their mobile phone via the application, as well as a simplified faceup version of $3 D$ matching so that they see the evolution of their treatment. Their involvement is thus rewarded, and their active participation in the treatment is recognized.

Patients also receive automatic notifications to remind them to take their photo test on time. It is a motivating tool which promotes the smooth progression of the treatment.

\section{The practitioner's communication tools}

Orthodontists can send a personalized message directly to patients' phone after consulting the DM patient record. A notification on the DM application icon then informs patients. All messages from the practitioners are retained on the patients' app. For children, a copy is sent by mail to the parents. This follow-up work by the practitioners builds a strong link with their patients, as the practitioners help stimulate their patients' motivation. This is either by empathic messages when a new device is installed or by messages of direct motivation when there is an observable reduction in the wearing of intermaxillary traction elastics visible on the monitoring curves.

Orthodontists can also remotely activate an emergency exam with the addition of specific instructions to be observed before the photographs are taken.

Some examples:

- Should there be any doubt as to the progression of treatment: "Could you please put on your elastics so that I can monitor their correct positioning? Thank you, Dr. Roisin."

- In case of poor hygiene: "I have noticed that you are not brushing enough; please make a little more effort, and I will check in again in 2 weeks. Thank you, Dr. Roisin."

\section{Sharing tools with colleagues}

Orthodontists may share patient monitoring files in read-only mode with the corresponding dental surgeons, periodontists, maxillofacial surgeons, or other professional correspondents. From the dashboard, they simply has to type in the practitioners' email, possibly with an associated message.

\section{CONCLUSION}

Orthodontic monitoring paves the way to a connected orthodontics, in line with the progress of modern orthodontic practices which include the following:
- A patient who is more invested in their treatment

- Interactive communication with patients outside of the office 
- A regular, frequent, precise, and individualized follow-up of dental movements and therapeutic actions

- The early detection of incidents and side effects leading to a reduction in complications.

Orthodontic monitoring controls treatments so as to optimize their treatment progress. This is done by empowering patients and by tracking the effects and undesirable incidents.
It is a remarkable efficiency factor. The accumulation of treatment data at a high frequency creates, for the first time, a huge database of dental movements that "threatens" to challenge our daily practices.

Conflict of interest: Dr. Roisin is the clinic director at Dental Monitoring. Dr. Brezulier and Professor Sorel declare that they have no conflict of interest with Dental Monitoring.

\section{BIBLIOGRAPHY}

1. Aggarwal J, Vemuri B, Chen Y, Medioni G. Range Image Understanding Object modelling by registration of multiple range images. Image Vis Comput 1992;10(3):145-155.

2. Blacher J, et al. Prise en charge de I'hypertension artérielle de l'adulte. Recommandations 2013 de la Société française d'hypertension artérielle. Ann Cardiol Angéiologie 2013;62(3):132-138.

3. Blevins T. Value and utility of self-monitoring of blood glucose in non-insulin-treated patients with type 2 diabetes mellitus. Postgrad Med 2013;125(3):191-204.

4. Boringi M, et al. Knowledge and Awareness of Teledentistry among Dental Professionals A Cross Sectional Study. J Clin Diagn Res 2015;9(8):ZC41-44.

5. Canadian Hypertension Education Program. The 2008 Canadian Hypertension Education Program recommendations: the scientific summary - an annual update. Can J Cardiol 2008;24(6):447-452.

6. Christiansen S, Klötzer J-P. [Telemedicine for heart failure, diabetes and chronic obstructive pulmonary disease]. Versicherungsmedizin Hrsg Von Verband LebensversicherUnternehm EV Verband Priv Krankenversicher EV 2016;68(1):20-22.

7. Daniel SJ, Kumar S. Teledentistry: a key component in access to care. J Evid-Based Dent Pract 2014;14 Suppl:201-208.

8. Daniel SJ, Wu L, Kumar S. Teledentistry: a systematic review of clinical outcomes, utilization and costs. J Dent Hyg 2013;87(6):345-352.

9. De Greeff A, Shennan AH. Validation of the Tensoval Duo Control II blood pressure monitor for clinic use and self-measurement according to the British Hypertension Society protocol and the European Society of Hypertension International Protocol Revision 2010. Blood Press Monit 2013;18(3):161-166.

10. Dutagaci $H$, Cheung CP, Godil A. Evaluation of $3 D$ interest point detection techniques via human- generated ground truth. Vis Comput 2012;28(9):901-917.

11. Eckhoff RP, Kizakevich PN, Bakalov V, Zhang Y, Bryant SP, Hobbs MA. A Platform to Build Mobile Health Apps: The Personal Health Intervention Toolkit (PHIT). JMIR MHealth UHealth. 2015;3(2):e46. 
12. Estai M, et al. End-user acceptance of a cloud-based teledentistry system and Android phone app for remote screening for oral diseases. J Telemed Telecare 2015 [Epub ahead of print]

13. Estai M, et al. A proof-of-concept evaluation of a cloud-based store-and-forward telemedicine app for screening for oral diseases. J Telemed Telecare 2015 [Epub ahead of print]

14. Glynn LG, Murphy AW, Smith SM, Schroeder K, Fahey T. Self-monitoring and other non-pharmacological interventions to improve the management of hypertension in primary care: a systematic review. Br J Gen Pract 2010;60(581):e476-488.

15. Greene JA. Do-lt-Yourself Medical Devices-Technology and Empowerment in American Health Care. N Engl J Med 2016;374(4):305-308.

16. Haddad AE, Bönecker M, Skelton-Macedo MC. Research in the field of health, dentistry, telehealth and teledentistry. Braz Oral Res 2014;28.

17. Haute Autorité de Santé - Éducation thérapeutique du patient [Internet]. 2007 Disponible sur: http://www. has-sante.fr/portail/jcms/c_602687/fr/education-therapeutique-du-patient

18. Haute Autorité de Santé - L'éducation thérapeutique dans la prise en charge des maladies chroniques: Analyse économique et organisationnelle [Internet]. 2007 Disponible sur: http://www.has-sante.fr/portail/jcms/r_1499485/fr/leducation-therapeutique-dans-laprise-en-charge-des-maladies-chroniques-analyse-economique-et-organisationnelle?xtm$\mathrm{C}=\& \mathrm{xtcr}=6$

19. How to measure home blood pressure: Recommendations for healthcare professionals and patients. - PubMed - NCBI [Internet]. Disponible sur: http://www.ncbi.nlm.nih.gov/ pubmed/27051984

20. Imai $Y$, et al. Japanese society of hypertension (JSH) guidelines for self-monitoring of blood pressure at home. Hypertens Res 2003;26(10):771-782.

21. Jia Y, et al. Caffe: Convolutional Architecture for Fast Feature Embedding. ArXiv14085093 Cs [Internet]. 20 juin 2014; Disponible sur: http://arxiv.org/ abs/1408.5093

22. Kolb H, Kempf K, Martin S, Stumvoll M, Landgraf R. On what evidence-base do we recommend self-monitoring of blood glucose? Diabetes Res Clin Pract 2010;87(2):150-156.

23. Kruger E, Estai M, Tennant M. Perceptions of Australian dental practitioners about using telemedicine in dental practice. Br Dent J 2016;220(1):25-29.

24. Lacroix A. Quels fondements théoriques pour l'éducation thérapeutique? Santé Publique 2007;19(4):271-282.

25. Lee JM, Hirschfeld E, Wedding J. A Patient-Designed Do-lt-Yourself Mobile Technology System for Diabetes: Promise and Challenges for a New Era in Medicine. JAMA 2016;315(14):1447-1448.

26. Li L, Zhang X, Yan C, Liang Q. Validation of the Oregon Scientific BPU 330 for self-monitoring of blood pressure according to the International Protocol. Vasc Health Risk Manag 2008;4(5):1121-1125.

27. Lindeberg T. Image Matching Using Generalized Scale-Space Interest Points. In: Kuijper A, Bredies K, Pock T, Bischof H, éditeurs. Scale Space and Variational Methods in Computer Vision [Internet]. Springer Berlin Heidelberg; 2013 [cité 8 mai 2016]. p. 355-367. (Lecture Notes in Computer Science). Disponible sur: http://linkspringer.com/chapter/ 10.1007/978-3-642-38267-3_30 
28. Mackillop LH, et al. Trial protocol to compare the efficacy of a smartphone-based blood glucose management system with standard clinic care in the gestational diabetic population. BMJ Open. 2016;6(3):e009702.

29. Mariño R, Tonmukayakul U, Manton D, Stranieri A, Clarke K. Cost-analysis of teledentistry in residential aged care facilities. J Telemed Telecare 2015; [Epub ahead of print]

30. McLaren SW, Kopycka-Kedzierawski DT. Compliance with dental treatment recommendations by rural paediatric patients after a live-video teledentistry consultation: A preliminary report. J Telemed Telecare 2016;22(3):198-202.

31. McManus RJ, Mant J, Bray EP, Holder R, Jones MI, Greenfield S, et al. Telemonitoring and self- management in the control of hypertension (TASMINH2): a randomised controlled trial. The Lancet 2010;376(9736):163-172.

32. O'Brien E, et al. European Society of Hypertension International Protocol revision 2010 for the validation of blood pressure measuring devices in adults. Blood Press Monit 2010;15(1):23-38.

33. Pickering TG, White WB. When and how to use self (home) and ambulatory blood pressure monitoring. J Am Soc Hypertens 2010;4(2):56-61.

34. Ramesh N, et al. Teledentistry: knowledge and attitudes among dentists in Udaipur, India. Oral Health Dent Manag 2013;12(3):138-144.

35. Saxon LA. Mobile Health Application Solutions. Circ Arrhythm Electrophysiol 2016;9(2): e002477.

36. Schiff A. Telemonitoring - or better follow-up? The Lancet 2010;376(9754):1737.

37. Scorpiglione $\mathrm{N}$, et al. Epidemiology and determinants of blood glucose self-monitoring in clinical practice. Diabetes Res Clin Pract 1996;34(2):115-125.

38. Sieverdes JC, Treiber F, Jenkins C. Improving diabetes management with mobile health technology. Am J Med Sci 2013;345(4):289-295.

39. Sipiran I, Bustos B. Harris 3D: a robust extension of the Harris operator for interest point detection on 3D meshes. Vis Comput 2011;27(11):963-976.

40. The SMBG International Working Group. Selfmonitoring of blood glucose in type 2 diabetes: An inter-country comparison. Diabetes Res Clin Pract 2008;82(3):e15-e18.

41. Vaïsse B, Mourad J-J, Girerd X, Hanon O, Halimi J-M, Pannier B. Enquête FLAHS 2012: la pratique de l'automesure tensionnelle en France et son évolution depuis 2010. Ann Cardiol Angéiologie 2013;62(3):200-203.

42. Varanauskiene E. Can blood glucose self-monitoring improve treatment outcomes in type 2 diabetes? 